\title{
Quantum Hierarchical Clustering Algorithm Based on the Nearest Cluster Centroids Distance
}

\author{
Fengbo Kong, Hong Lai, and Hailing Xiong
}

\begin{abstract}
It is getting harder to deal with the large data sets by the classical hierarchical clustering algorithm, so we propose an efficient quantum hierarchical clustering algorithm, in which the quantum bit (qubit) is used to represent the data point in the space. For quantum entanglement, the distance between two data points is calculated through adding an auxiliary particle to construct the entangled state. Then a projective measurement is performed on the auxiliary particle alone. The distance between two points is acquired by the projective measurement. We use the distance of the cluster centroids as a measure of similarity between clusters. Also, based on the principle of the minimum cluster centroids distance, the nearest two clusters are merged. We aim at improving time and space complexity and effect of the clustering of the hierarchical clustering algorithm.
\end{abstract}

Index Terms-Large data, hierarchical clustering, qubit, entangled states.

\section{INTRODUCTION}

Nowadays, on the one hand, the data generated by mankind increase rapidly beyond our imagination. On the other hand, the size of the classic computer chip is difficult to become smaller as the post-Moore era ends, so further improvements in the calculation speed seem to be impossible. Scientists predicted big data analysis such as machine learning, natural language processing and pattern recognition will face more severe challenges in the future [1]-[3].

As one of the important research contents of machine learning, pattern recognition and so on, the clustering analysis plays an extremely important role in identifying the internal structure of data. The clustering analysis usually can be divided into five categories, partitioning-based approach, hierarchical approach, density-based approach, grid-based approach, and model-based approach [4], [5]. Hierarchical clustering analysis is one of the pillars of clustering analysis

Manuscript received August 21, 2017; revised October 17, 2017. This work was financially supported by the National Natural Science Foundation of China (41271292, 61702427), the Fundamental Research Funds for the Central Universities (XDJK2016C043), the Doctoral Program of Higher Education (SWU115091), and the 1000-Plan of Chongqing by Southwest University (SWU116007).

Fengbo Kong and Hailing Xiong are with College of Computer and Information Science, Southwest University, Chongqing 400715, China (email: kfb_wavelet@163.com,xionghlt@swu.edu.cn).

Hong Lai is with College of Computer and Information Science and Centre for Research and Innovation in Software Engineering (RISE), Southwest University, Chongqing 400715, China (e-mail: hlai@swu.edu.cn). in practical application [6]-[9] because it is simple and easy to implement. However, in the era of big data, the development and application of most clustering algorithms are constrained by the high time and space complexity. Hierarchical clustering analysis also has the same dilemma. Although hierarchical clustering analysis is simple, it seems to be too slow for the large data sets. The time complexity of at least $O\left(n \log _{2} n\right)$ is required, where $n$ is the number of data points. However, quantum computing can provide a good solution for the low efficiency of the clustering analysis algorithm.

In recent decades, quantum computing has made amazing achievements [10]-[13]. Quantum coherence and quantum entanglement are used to quantum computing, which making quantum computing has a unique way of calculating that is completely different from the classical computation. Moreover, the parallelism of quantum computing shows remarkable superiority that classic computing cannot catch up. In 1989, Deutsch first proposed the Deutsch quantum algorithm [10], which is used to solve the $n$-bit Deutsch problem. This quantum algorithm is a good demonstration of the parallelism of quantum computers for the first time. It also brings the inspiration for the quantum computing research. The efficiency of quantum computer is far faster indeed than that of classic computer in solving some problems. After that, Shor [11], [12] and Grover [13] showed the advantages of quantum computing in solving some of the classical problems respectively, such as RSA, DES, AES. Since then, the Algorithm quantization sets off a research boom in the international academia, and the quantum computing provides a new way of accelerating the classic algorithms.

In the past ten years, some scholars carried out some preliminary researches about combinatorial machine learning and quantum algorithms [14]-[17]. Aïmeur et al. [18] first proposed learning in quantum states in 2006, and they put forward a quantum dichotomy algorithm. The fidelity of quantum states is used to determine the quantum state similarity. In 2008, a learning model of quantum state was raised by Gambs et al. [19], which is used to classify an unknown quantum state. In 2013, Lloyd et al. [20] proposed a supervised and unsupervised quantum machine learning. After that, more and more quantum machine learning algorithms have been presented [21]-[24]. In 2015, Cai et al. [25] implemented an experiment, based on entangled quantum machine learning. Although the above quantum machine learning algorithms use the quantum way to achieve the classification or clustering analysis, most of them focused on such case as dividing all objects into two clusters. As for the case of dividing into any number of clusters, it still needs to be further studied. Thus, we propose a quantum hierarchical clustering algorithm based on the classical 
hierarchical clustering analysis. This algorithm has three improvements: (1) the qubit is applied to represent the data point in the space, (2) the distance between the points is calculated by introducing an auxiliary particle to construct the quantum entangled states, and (3) the minimum cluster centroids distance between clusters is used as the merging rule, instead of the minimum distance of two points between clusters.

\section{ClassicAl HiERARCHICAL CluStering ANALYSIS}

In the hierarchical clustering analysis, there are two fundamental types of hierarchical clustering analysis, agglomerative hierarchical clustering algorithm based on bottom-up merging and divisive hierarchical clustering algorithm with top-down splitting [26]. Strategies for the two types are as follow:

- Agglomerative clustering: it is a bottom-up approach. Each object is its own initial cluster. As one move up the hierarchy, pairs of clusters are merged. Until all objects are in the same cluster, it ends.

- Divisive clustering: it is a top-down approach. All objects start in one cluster. Then one cluster is split into two clusters recursively as one move down the hierarchy. Until all clusters only contain one object respectively, it ends.

Both this algorithm are exactly reverse of each other. So in this paper, we focus on the quantization of the agglomerative hierarchical clustering algorithm in detail. The specific algorithm steps of the classic agglomerative hierarchical clustering are as follows:

Input: $n$ data points, the number of terminating clusters $k$; Output: $k$ clusters;

(1) Let each data point be an initial cluster, respectively;

(2) Compute the similarity of clusters between pairs of clusters and find the nearest two clusters;

(3) Merge the nearest two clusters to generate a new set of clusters;

(4) Repeat the second and the third steps until the number of defined clusters $k$ is reached.

Fig. 1 describes the processing of an example about the agglomerative hierarchical clustering method and the divisive one. The data sets contain five objects $\{a, b, c, d, e\}$.

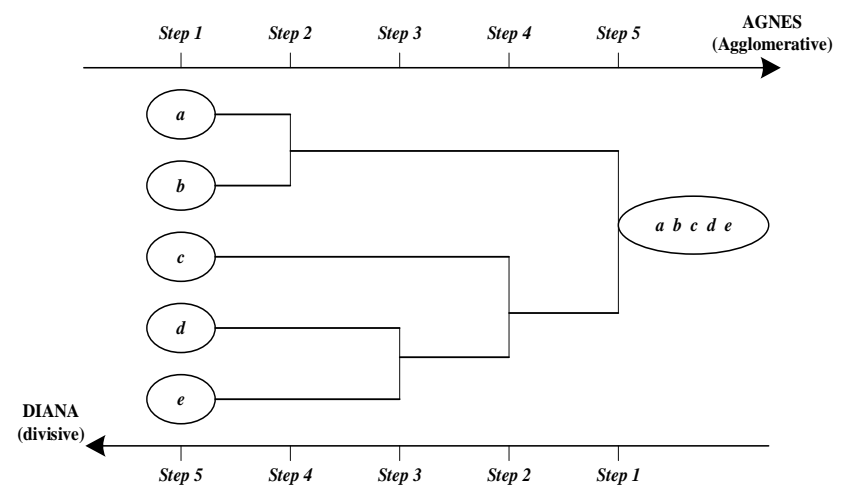

Fig. 1. Aggregation and divisive hierarchical clustering of data objects $\{a$, b, c, d, e\}.

According to the number of clusters by user requirements, the data sets can be divided at different hierarchies. For example, the user requests the five objects to be divided into three clusters, then the aggregation hierarchical clustering algorithm just needs to work to the third step of the Fig. 1. The advantages of the algorithm have been listed in the above section, but the computational complexity still restricts its application scope in large volumes of data. Also, effect of the clustering is not ideal to a certain extent.

\section{QUANTUM HIERARCHICAl ClUSTERING AlgORITHMS}

With the increase of the data sets, the time and space complexity of the classical hierarchical clustering analysis mostly exponentially rise. Luckily, its time and space complexity can be greatly reduced by the combination of quantum computing and the classical algorithms.

\section{A. Quantum Representation of Data Points in the Space and the Distance}

The biggest difference between our algorithm and the classical hierarchical clustering is the representation of data points and the calculation of distance in the space.

1) Quantum representation of the data points in the space

Data points in space are vectors. Quantum states can be used to represent data points in space, such as data point $\vec{x}_{i}$, which can be represented by quantum state $\vec{x}_{i}=\left|x_{i}\right|\left|x_{i}\right\rangle$, where $\left|x_{i}\right\rangle$ represents qubit. For example, when the vector is four dimensions, $\left|x_{i}\right\rangle$ can be expressed as:

$$
\left|x_{i}\right\rangle=\alpha_{0}|00\rangle+\alpha_{1}|01\rangle+\alpha_{2}|10\rangle+\alpha_{3}|11\rangle
$$

where $|00\rangle,|01\rangle,|10\rangle,|11\rangle$ are the calculation basis, $\left|\alpha_{\mathrm{i}}\right|^{2}$ $(i=0,1,2,3)$ is the probability that the corresponding calculation basis is obtained after the measurement, all coefficients $\alpha_{i}(i=0,1,2,3)$ are satisfied the condition of $\sum_{i=0}^{3}\left|\alpha_{i}\right|^{2}=1$

2) Quantum representation of the distance between two data points

According to the above description, we could get the distance between the two points of the quantum expression of $D$ :

$$
\begin{aligned}
D & =\left|\overrightarrow{x_{i}}-\overrightarrow{x_{j}}\right|=\sqrt{\left|\overrightarrow{x_{i}}-\overrightarrow{x_{j}}\right|^{2}} \\
& =\sqrt{\left(| x _ { i } | \left\langlex_{i}|-| x_{j}\left|\left\langle x_{j}\right|\right)\left(\left|x_{i}\right|\left|x_{i}\right\rangle-\left|x_{j}\right|\left|x_{j}\right\rangle\right)\right.\right.}
\end{aligned}
$$

The core idea of the quantum algorithm is to use the coherence of the quantum state, so that the result of the satisfying condition is enhanced while the result of the conditional dissatisfaction is weakened. Thus, the result of the satisfying condition occurs at a high probability after the measurement. Quantum entanglement reflects the coherence, the probability and the spatial nonlocalization of quantum states. Therefore, an auxiliary qubit is added to construct the quantum entangled state between the two data points. Through the measurement of the entangled state, we obtain the relation among the probability, the Euclidean distance and the inner product. Then we could get the distance of the 
two data points. The detailed computing steps of distance $D$ are described in the next subsection.

\section{B. Algorithms Description}

In the subsection, we describe the concrete steps of the quantum hierarchical clustering algorithm as follows. There is a data set $X$ that contains $N d$-dimensional data, where $d=2^{n}$.

Input: $N$ quantum states of $n$ qubits, user-specified cluster numbers $k$ ( $k$ is much smaller than $n)$;

Output: $k$ quantum superposition states (i.e., $k$ clusters)

(1) Each point as an initial cluster, that is, each quantum state as an initial cluster, and the initial cluster centroids is itself for each point. So, let $t=0$, where $t$ denotes the times of merger,

(2) Calculate the distances of cluster centroids between pairs of clusters. According to the minimum cluster centroids distance rule, the two nearest clusters are merged into the same cluster. Then, $t=t+1$,

(3) Calculate the cluster centroids of the clusters after reclassification and update them. $\overrightarrow{c_{r}}$ is the cluster centroids of the cluster $r, \overrightarrow{c_{r}}=\left|c_{r}\right|\left|c_{r}\right\rangle=\frac{1}{n_{r}} \sum_{i=1}^{n_{r}}\left|x_{i}\right|\left|x_{i}\right\rangle$, where $n_{r}$ is the number of data points in the cluster $r, x_{i}$ is the data point in the cluster $r$,

(4) It ends until $t$ satisfies $t=n-k$. Otherwise, repeat the second and the third steps.

In the second step of the quantum hierarchical clustering algorithm, the operation of calculating the distance $D$ as follows:

(1) The way of calculating the distance between two points is to add an auxiliary qubit between the two points $\overrightarrow{x_{i}}$ and $\overrightarrow{x_{j}}$. They formed an entangled state:

$$
|\varphi\rangle=\frac{1}{\sqrt{2}}\left(|0\rangle_{\text {anc }}\left|x_{i}\right\rangle+|1\rangle_{a n c}\left|x_{j}\right\rangle\right)
$$

Constructing the entangled state of (3) could be obtained by querying QRAM. The QRAM consists of an address register and an output register. The address register contains an address of the superposition of $\sum_{j} p_{j}|j\rangle_{a}$ (where $j$ has not any relationship with the $j$ of $x_{j}$ ). The output register contains the information superimposed state $\sum_{j} p_{j}|j\rangle_{a}\left|D_{j}\right\rangle_{d}$ associated with the address register. For example, the QRAM output register and the address register are considered as a function $f$ and the input of the function $f$ respectively. When the address is $\frac{1}{\sqrt{2}}(|0\rangle+|1\rangle)$ and the data is $|\mathrm{D}\rangle_{\mathrm{d}}=\left|x_{i}\right\rangle+\left|x_{j}\right\rangle$, the output is $f\left(\frac{|0\rangle}{\sqrt{2}}, \frac{|1\rangle}{\sqrt{2}}\right)=\frac{1}{\sqrt{2}}\left(|0\rangle\left|x_{i}\right\rangle+|1\rangle\left|x_{j}\right\rangle\right)$, i.e., the required entangled state.

(2) Take a projective measurement of $|\varphi\rangle$ to observe if the measurement results on $|\phi\rangle$ :

$$
|\phi\rangle=\left(\left|x_{i}\right||0\rangle-\left|x_{j}\right||1\rangle\right) / \sqrt{\left|x_{i}\right|^{2}+\left|x_{j}\right|^{2}}
$$

In order to generate $|\phi\rangle$, we carry out unitary transformation $e^{-i H t}$ for state $\frac{1}{\sqrt{2}}(|0\rangle-|1\rangle) \otimes|0\rangle$, where
$\mathrm{H}=\left(\left|\overrightarrow{x_{i}}\right||0\rangle\left\langle 0|+| \overrightarrow{x_{j}}|| x_{j}\right\rangle\right) \otimes \sigma_{x} \quad$. The result of the transformation as follows:

$$
\begin{aligned}
& \frac{1}{\sqrt{2}} \cos \left(\left|\overrightarrow{x_{i}}\right| t\right)|0\rangle-\cos \left(\left|\overrightarrow{x_{j}}\right| t\right)|1\rangle \otimes|0\rangle S \\
& -\frac{i}{\sqrt{2}} \sin \left(\left|\overrightarrow{x_{i}}\right| t\right)|0\rangle-\sin \left(\left|\overrightarrow{x_{j}}\right| t\right)|1\rangle \otimes|1\rangle
\end{aligned}
$$

Select an appropriate $t$, which meets the condition of $\left|\overrightarrow{x_{i}}\right| t,\left|\overrightarrow{x_{j}}\right| t \ll 1$. Then (5) is computed, we obtain $|\phi\rangle$ by the probability of $\frac{1}{2}\left(\left|\overrightarrow{x_{i}}\right|^{2}+\left|\overrightarrow{x_{j}}\right|^{2}\right) t^{2}$. Repeat a projective measurement on $|\varphi\rangle$, we obtain $|\phi\rangle$ with the probability of $p$ :

$$
p=p(|\phi\rangle)=\left\langle\varphi\left|M_{\phi}^{+} M_{\phi}\right| \varphi\right\rangle
$$

Then, according to $M_{\phi}=|\phi\rangle\langle\phi|$, (6) can be rewritten as follows:

$$
\begin{aligned}
p= & p(|\phi\rangle)=\langle\varphi \mid \phi\rangle\langle\phi \mid \varphi\rangle \\
= & \frac{\left\langle x_{i}\right|\langle 0|+\left\langle x_{j}\right|\langle 1|}{\sqrt{2}} \cdot \frac{\left|x_{i}\right||0\rangle-\left|x_{j}\right||1\rangle}{\sqrt{\left|x_{i}\right|^{2}+\left|x_{j}\right|^{2}}} \\
& \cdot \frac{\left|x_{i}\right|\left\langle 0|-| x_{j}\right|\langle 1|}{\sqrt{\left|x_{i}\right|^{2}+\left|x_{j}\right|^{2}}} \cdot \frac{|0\rangle\left|x_{i}\right\rangle+|1\rangle\left|x_{j}\right\rangle}{\sqrt{2}} \\
= & \frac{\left(| x _ { i } | \left\langlex_{i}|-| x_{j}\left|\left\langle x_{j}\right|\right) \cdot\left(\left|x_{i}\right|\left|x_{i}\right\rangle-\left|x_{j}\right|\left|x_{j}\right\rangle\right)\right.\right.}{2\left(\left|x_{i}\right|^{2}+\left|x_{j}\right|^{2}\right)}
\end{aligned}
$$

Combined (2) with (7), the distance between $\overrightarrow{x_{i}}$ and $\overrightarrow{x_{j}}$ is obtained as follows:

$$
D=\sqrt{2 p\left(\left|x_{i}\right|^{2}+\left|x_{j}\right|^{2}\right)}
$$

Expand (7):

$$
\begin{aligned}
p & =p(|\phi\rangle) \\
& =\frac{\left|x_{i}\right|^{2}-\left|x_{i}\right|\left|x_{j}\right|\left\langle x_{i} \mid x_{j}\right\rangle-\left|x_{i}\right|\left|x_{j}\right|\left\langle x_{j} \mid x_{i}\right\rangle+\left|x_{j}\right|^{2}}{2\left(\left|x_{i}\right|^{2}+\left|x_{j}\right|^{2}\right)}
\end{aligned}
$$

From (9), we obtain the inner product expression between $\left|x_{i}\right\rangle$ and $\left|x_{j}\right\rangle$ as follows:

$$
\left\langle x_{i} \mid x_{j}\right\rangle+\left\langle x_{j} \mid x_{i}\right\rangle=(1-2 p)\left(\left|x_{i}\right|^{2}+\left|x_{j}\right|^{2}\right) /\left(\left|x_{i}\right|\left|x_{j}\right|\right)
$$

In the third step of the quantum hierarchical clustering algorithm, the cluster centroids of each cluster are calculated. Assume that there are $n_{i}$ points in the $i$-th clusters, i.e., $\left\{x_{1}, x_{2}, \ldots, x_{n i}\right\}$, we could calculate the cluster centroids:

$$
\frac{x_{1}+x_{2}+\cdots+x_{n_{i}}}{n_{i}}=\frac{\left|x_{1}\right|\left|x_{1}\right\rangle+\cdots+\left|x_{n_{i}}\right|\left|x_{n_{i}}\right\rangle}{n_{i}}=\frac{1}{n_{i}} \sum_{k=1}^{n_{i}}\left|x_{k}\right|\left|x_{k}\right\rangle
$$

\section{ANALYSIS OF ALGORITHMS}

The quantum bit (qubit) is not yet clearly defined. Compared to the bits in the classic information, most 
scholars are accustomed to the qubits as the basic unit of quantum information. In the classical information, the classical bits have two states of 0 and 1 . A classical bit string consisting of 0 and 1 is encoded to express different classical information. In quantum information, the qubits are typically represented by two quantum states $|0\rangle$ and $|1\rangle$, then encoded with qubits consisting of $|0\rangle$ and $|1\rangle$. In the quantum world, a qubit, which continuously and randomly exists in any superposition state, is a vector in a twodimensional Hilbert space. The superposition state of a qubit can be expressed by the formula $|\Psi\rangle=\alpha|0\rangle+\beta|1\rangle$, where both $\alpha$ and $\beta$ are complex number and satisfy the condition of $|\alpha|^{2}+|\beta|^{2}=1$. The quantum superposition state is one of the key characteristics that make the quantum calculation different from the classical calculation. It is also the theoretical basis of quantum parallel computing [27]. For a register of $n$ qubits, the quantum superposition states of $n$ qubits can be represented by $|\Psi\rangle=\sum_{x=0}^{2^{n}-1} c^{n}|x\rangle$. Making calculation of this formula once is equivalent to making calculation of numbers at the same time, which makes the time complexity of the algorithm decrease substantially. In our algorithm, the time cost mainly depends on the distance calculation. The time complexity of the classical hierarchical clustering in calculating the distance between two $d$ dimensional vectors is $O(d)$. However, in the quantum world, only the time complexity of $O\left(\log _{2} d\right)$ was required in the same data sets [28]. From the time spend of calculating the distance of two $d$-dimensional data, we could no exaggeration to say that our algorithm greatly reduces the time cost. To save time often means that the more storage space will be used [29]-[31]. But in the quantum world, it is not the case.

For the space complexity, we take the information quantity expressed by two bits as an example to illustrate the space complexity analysis of our algorithm. In classic calculation, 00,01,10,11 are used to express four different messages respectively. The two bits of classic memory can only store one of the four states once, while the two qubits of quantum memory can store these four different states in the form of quantum superposition at the same time. It means that the storage capacity of the quantum computer increase exponentially, compared to the traditional computer on the condition of the same bits of the register. For example, for an $n$-qubit register, $2^{n}$ different numbers can be stored at the same time, and the $2^{n}$ different numbers are expressed in the same one quantum state. The space complexity of our algorithm mainly falls on the cost of the storage of data sets. Compared to the classic storage, $1 / 2^{n}$ the ability of the classic storage register is enough for our algorithm to store the same size of the data sets.

Due to the quantum parallel computing, the time and space complexity of our algorithm decrease sharply. Quantum parallel computing reflects the most important advantage of quantum computing. The classic computer computing speed and information processing capabilities cannot be compared with the quantum computer. Thus, the quantum hierarchical clustering algorithm is superior to the classical one in the time and space complexity through the above analysis. The advantages of our algorithm are more obvious, especially on large data sets.

\section{CONCLUSION}

In this paper, we have elaborated the hierarchical clustering analysis based on quantum computations. Then we have proposed the quantum calculation method of the distance between two data points. Finally, we analyzed the time and space complexity of the proposed algorithm. It shows that the quantum hierarchical clustering algorithm could bring an exponential speed-up over classical algorithms. Moreover, considering the shortcoming that classical hierarchical clustering cannot revoke the merging operation of the previous step, we use the principle of the minimum cluster centroids distance replace the principle of the minimum data points distance. It makes effect of the clustering much better than the classical one. Of course, this alternative strategy is not perfect. But we will further study how to overcome the lack of the quantum hierarchical clustering algorithm well in the follow-up work.

\section{REFERENCES}

[1] A. Labrinidis and H. V. Jagadish, "Challenges and opportunities with big data," VLDB Endowment, 2012.

[2] J. Fan, F. Han, and H. Liu, "Challenges of big data analysis," National Science Review, vol. 1, no. 2, p. 293, 2014.

[3] D. Agrawal, P. Bernstein, E. Bertino et al., "Challenges and opportunities with big data," Community White Paper, United States, 2012.

[4] L. He, L. D. Wu, and Y. Z. Cai, "A survey of clustering algorithms in data mining," Application Research of Computers, vol. 24, no. 1, pp. 10-13, 2007

[5] Q. L. Hu, N. Y. Ye, and M. F. Zhu, "A survey of clustering algorithms in data mining," Computer \& Digtal Engineering, vol. 35, no. 2, pp. 17-20, 2007.

[6] C. M. Bishop and M. E. Tipping, "A hierarchical latent variable model for data visualization," IEEE Transactions on Pattern Analysis \& Machine Intelligence, vol. 20, no. 3, pp. 281-293, 1998.

[7] S. F. Tian, Artificial Intelligence and Knowledge Engineering, China Railway Publishing House, 1999.

[8] C. K. I. Williams, "A MCMC approach to hierarchical mixture modelling," in Proc. International Conference on Neural Information Processing Systems, MIT Press, 1999, pp. 680-686.

[9] C. Fraley, "Algorithms for model-based gaussian hierarchical clustering," Society for Industrial and Applied Mathematics, 1998.

[10] D. Deutsch, "Quantum computational networks," Proceedings of the Royal Society of London, vol. 425, pp. 73-90, 1989.

[11] P. W. Shor, "Algorithms for quantum computation: Discrete logarithms and factoring," in Proc. 1994 Symposium on Foundations of Computer Science, 2002, pp. 124-134.

[12] P. W. Shor, "Polynomial-time algorithms for prime factorization and discrete logarithms on a quantum computer," Society for Industrial and Applied Mathematics, 1997.

[13] L. K. Grover, "Quantum mechanics helps in searching for a needle in a haystack," Proceedings of Ccast Quantum Entanglement and Quantum Information, pp. 325-328, 1999.

[14] H. K. Lau, R. Pooser, G. Siopsis, and C. Weedbrook, "Quantum machine learning over infinite dimensions," Physical Review Letters, vol. 118, no. 8, 2017.

[15] Y. B. Sheng and L. Zhou, "Distributed secure quantum machine learning," Science Bulletin, 2017.

[16] M. R. Hush, "Machine learning for quantum physics," Science, vol. 6325, pp. 580-580, 2017.

[17] C. Carlo, H. Mark et al, Quantum Machine Learning: A Classical Perspective, 2017.

[18] E. Aïmeur, G. Brassard, and S. Gambs, "Machine learning in a quantum world," Lecture Notes in Computer Science, pp. 431-442, 2006.

[19] S. Gambs, "Quantum classification," Computer Science, 2008.

[20] S. Lloyd, M. Mohseni, and P. Rebentrost, "Quantum algorithms for supervised and unsupervised machine learning," Eprint Arxiv, 2013. 
[21] E. C. Behrman and J. E. Steck, "A quantum neural network computes its own relative phase," arXiv preprint arXiv: 1301.2808, 2013.

[22] P. Rebentrost, M. Mohseni, and S. Lloyd, "Quantum support vector machine for big data classification," Physical Review Letters, vol. 113, no. 13, p. 130503, 2014.

[23] N. Wiebe, A. Kapoor, and K. M. Svore, "Quantum nearest-neighbor algorithms for machine learning," Quantum Information and Computation, vol. 15, 2015.

[24] M. Schuld, I. Sinayskiy, and F. Petruccione, "Prediction by linear regression on a quantum computer," Physical Review A, 2016, vol. 94, no. 2, 022342 .

[25] X. D. Cai, D. Wu, Z. E. Su et al., "Entanglement-based machine learning on a quantum computer," Physical Review Letters, vol. 114 no. $11,2015$.

[26] J. Han, J. Pei, and M. Kamber, Data Mining: Concepts and Techniques, Elsevier, 2011.

[27] Y. Wang, "Research on clustering algorithm based on quantum theory," Zhejiang University of Technology, 2011.

[28] X. Y. Zhou, X. X. An, W. J. Liu, and F. G. Ji, "Quantum K-means Algorithm Based on the Minimum Distance," Journal of Chinese Computer Systems, vol. 38, no. 5, pp. 1059-1062, 2017.

[29] H. N. Qi, "Introduction to Computer," Science Press, 2014.

[30] Z. H. Zheng and X. M. Zheng, "Design and analysis of algorithms," TsingHua University Press, 2011.

[31] W. H. Wu and H. Sun, "Combinatorial math in programming," TsingHua University Press, 2005.

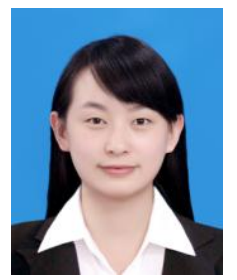

Fengbo Kong is currently a graduate student at College of Computer and Information Science, Southwest University, Chongqing, China. She received her bachelor's degree in computer science and technology at Nanjing University of Aeronautics and Astronautics in 2016. Her research interests include secret sharing, key agreement, quantum key distribution, quantum secret sharing.

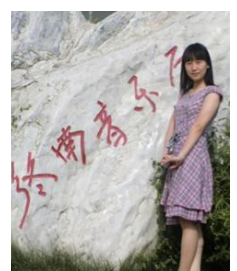

Hong Lai is currently an associate professor at College of Computer and Information Science, Southwest University, Chongqing, China. Dr Lai received her $\mathrm{PhD}$ from Macquarie University and Beijing University of Posts and Telecommunications in 2015. Her current research interests lie in the areas of secret sharing, key agreement, quantum key distribution, quantum secret sharing, quantum direction secure communication, quantum private queries and so on.

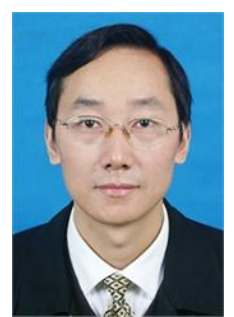

Hailing Xiong is currently a professor at College of Computer and Information Science, Southwest University, Chongqing, China. Prof. Xiong received his bachelor degree in agriculture at Southwest Agricultural University in 1994, the master degree in computer applications at Southwest Agricultural University in 2003, and then received the Doctor degree in soil science from Southwest University in 2007. He was a visiting scholar at University of California Riverside in 2010. His research interests are database and intelligent information processing, cellular automata theory and its applications. 